\title{
A Communication Theoretical Modeling of Axonal Propagation in Hippocampal Pyramidal Neurons
}

\author{
Hamideh Ramezani, Student Member, IEEE and Ozgur B. Akan, Fellow, IEEE
}

\begin{abstract}
Understanding the fundamentals of communication among neurons, known as neuro-spike communication, leads to reach bio-inspired nanoscale communication paradigms. In this work, we focus on a part of neuro-spike communication, known as axonal transmission, and propose a realistic model for it. The shape of the spike during axonal transmission varies according to previously applied stimulations to the neuron and these variations affect the amount of information communicated between neurons. Hence, to reach an accurate model for neurospike communication, the memory of axon and its effect on the axonal transmission should be considered, which are not studied in the existing literature. In this work, we extract the important factors on the memory of axon and define memory states based on these factors. We also describe the transition among these states and the properties of axonal transmission in each of them. Finally, we demonstrate that the proposed model can follow changes in the axonal functionality properly by simulating the proposed model and reporting the root mean square error between simulation results and experimental data.
\end{abstract}

Index Terms-Nanoscale communication, Molecular communication, Biomedical communication, Neuro-spike communication, Axonal functionality, Spike amplitude variation.

\section{INTRODUCTION}

$\mathbf{N}$ ANOMACHINES, i.e., nanoscale devices, need to establish network with each to do complex tasks. Among different communication paradigms existing in the literature for nanonetworking, molecular communication is the most promising one as a result of its biocompatibility and biostability [1]. Communication among neurons, i.e., neuro-spike communication, is one of the most sophisticated and practical mechanisms for molecular communication [2]. Hence, proposing a proper model for this communication is a significant step in finding a realistic model for nanonetworking [3].

A simplified schematic of communication between two neurons is depicted in Fig. 1. This communication contains three phases as outlined below:

1) Spike generation: In this phase, if the received stimulations from dendrites of the input neuron are strong enough, the neuron fires a molecular impulse known as action potential (AP) or spike.

2) Axonal transmission: The spikes pass through the axon of the neuron until reaching the axonal terminals.

Authors are with the Next-generation and Wireless Communications Laboratory (NWCL), Department of Electrical and Electronics Engineering, Koc University, Istanbul, Turkey (e-mail: \{hramezani13, akan\}@ku.edu.tr).

O. B. Akan is also with Electrical Engineering Division, Department of Engineering, University of Cambridge, UK (e-mail: oba21@cam.ac.uk).

This work was supported in part by ERC project MINERVA (ERC-2013CoG \#616922), and EU project CIRCLE (EU-H2020-FET-Open \#665564).

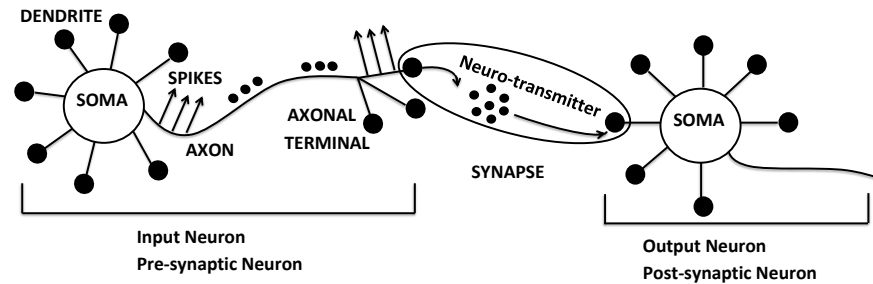

Fig. 1. Neural anatomy of neuro-spike communication.

3) Synaptic propagation: Arrival of a spike to the axonal terminals potentiates the release of vesicles, which are groups of chemical substances known as neurotransmitter, to the synapse, i.e., the gap between the pre- and postsynaptic neurons. If a vesicle releases, its neurotransmitters diffuse through the synapse. If these neurotransmitters reach receptors of the post-synaptic neuron, they bind to these receptors and stimulate the output neuron.

Spikes were considered as all or none for a long time, hence the existing studies in the literature on modeling neuro-spike communication [2], [4]-[6] mainly focused on the synaptic propagation and spike generation. However, with the use of direct recording from axons, variations in the shape of spikes during axonal transmission are recorded recently in different types of neurons such as hippocampal mossy fibers and pyramidal neurons [7]-[10]. A consequent result of the spike shape variations is change in the release of neurotransmitters to the synapse [10]-[12], which affects the amount of stimulation of the target cell [13]. Hence, to propose an accurate model for functionality of neurons, variations in the shape of the spike during axonal transmission should be considered.

The objective of this work is deriving a realistic channel model, which will, for the first time in the literature, incorporate the variations in the amplitude of spikes during axonal transmission from communication perspective. We select the pyramidal neurons in Cornu Ammonis (CA) area of hippocampus location in the brain for our work, since this area is heavily investigated in physiology, which provides experimental data for our work, and is a part of learning and memory in the brain. In this work, we use reported experimental data in the physiology literature to propose a model for effects of axonal transmission on the spike amplitude.

Based on the physiology literature, the axonal transmission depends on the previously fired spikes. Hence, we model the axon as a system with memory. For this aim, we extract the effective parameters in the memory of axon at first. Then, we define different states for the axonal functionality based 
on these parameters. Moreover, we provide the details of the transition among the proposed memory states and model the axonal functionality in each state. Finally, we reveal the accuracy of the proposed model by simulating it and comparing its results with experimental data.

The remainder of this paper is as follows. In Section II, we review the basics of axonal transmission and the existing communication model for it. In Section III, we introduce a realistic channel model for axonal transmission of hippocampal pyramidal neurons based on the physiological data. In Section IV, we compare results of the proposed model, experimental data and the existing model in the literature. We conclude our work and discuss open issues and future work in Section V.

\section{BACKGROUND}

In this section, we review the existing physiological studies on axonal transmission first. Then, we overview the existing communication channel model for axonal transmission.

\section{A. Neural Signaling}

The variations in the shape of the spike during axonal transmission were observed by applying two kinds of stimulations, (i) paired pulse stimulation, which is used to extract information about the refractory period of the neuron, i.e., the time that it does not fire any spike after firing one, and (ii) train stimulation, which is used to extract changes in the shape of the spike when the neuron is sufficiently depolarized.

In [9], [14], [15], hippocampal pyramidal neurons are stimulated by paired pulse stimulations with different inter pulse intervals (IPIs) varying between 1 and $500 \mathrm{~ms}$. The axon acts as a low-pass filter in response to these stimulations [16].

In [8], [9], [17], [18], trains of high frequency stimulation (HFS) with frequencies between 50 and $200 \mathrm{~Hz}$ are applied to hippocampal pyramidal neurons. Although pyramidal cells of CA1 can still fire during prolonged HFS, their axonal response to these spike trains weakens until the end of stimulation, and they may fail to respond at the end. Thus, prolonged HFS does not affect the spike generation phase, however, it changes the axonal functionality [17]. Based on experiments on pyramidal neurons of CA1 and CA3, applying the prolonged HFS increases the probability of failure in passing the spike through the axon. It also increases the delay and duration of evoked spike and decreases its amplitude [18]. However, as a result of the lack of information about changes in the width and delay of spikes, we only concentrate on spike amplitude variations.

After terminating HFS, the neuron passes two phases of recovery, one fast and frequency-dependent and another slow and frequency-independent. In Fast recovery, amplitude of the response recovers quickly, but the complete recovery occurs in the second recovery phase, i.e., Slow recovery. Moreover, during Fast recovery, refractory period of the neuron can increase based on the amount of suppression of axonal functionality and the duration of the time rest after HFS termination [8].

\section{B. Related Work}

Only one communication-theoretical channel model exists in the literature for axonal transmission [2], which is based on
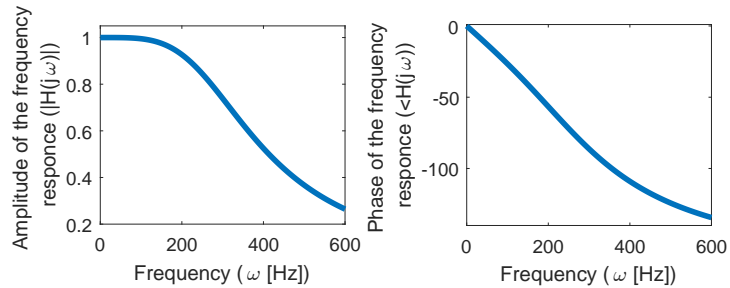

Fig. 2. Frequency response of the modified Butterworth filter [2].

[14], [16] and the assumption that the spike shape is fix. This channel model contains serial connection of following blocks.

1) Spike to rectangular pulse converter: In this block, impulses are converted to rectangular pulses such that when a spike comes, the pulse level changes and it remains there until the next spike.

2) Low-pass filter: The rectangular pulses are passed through a modified version of the second-order Butterworth filter with the frequency response given by

$$
H(j w)=\frac{a}{-w^{2}+j b w+a}[u(j w)-u(j w-c)],
$$

where $u($.$) is the Heaviside step function and the pa-$ rameters are selected as $a=314.15^{2}, b=444.28$, and $c=628.3$ [2]. The frequency response of this filter is depicted in Fig. 2.

3) Spike generator: In this block, filtered pulses with lowpass filter are converted to impulses.

The only effect of this filter is canceling spikes with small IPI based on the frequency response of the low-pass filter. However, based on new experiments [8], [9], the shape of an action potential may also change during axonal transmission according to the history of the neuron.

\section{Modeling Axonal Functionality}

The aim of this study is deriving the response of the axon to the stimulation, which depends on its memory from previously applied stimuli. Hence, we model the axon by a system with memory whose input is the IPI between the current and the previous stimulus, $\Delta t$, and its output is the amplitude of the axonal response to the stimulation, i.e., $A$. We have three tasks to model axonal functionality, (i) finding the important parameters for the axonal memory and defining memory states, (ii) deriving the relation of the output and the memory parameters in each memory state, and (iii) modeling the transition between memory states. These tasks are done by utilizing the existing experimental reports in the literature.

\section{A. Axonal Memory}

We model memory of the axon with following parameters:

- $A_{p}$, normalized amplitude of the latest evoked spike before arrival of the current stimulus. Normalized amplitude is the ratio of the amplitude of the evoked spike to the amplitude of the baseline spike, i.e., response of the neuron to a single stimulus after a long duration of not receiving any stimulation. Hence, the amplitude 
of the baseline spike does not depend on the memory, however, it varies for different type of neurons as a results of differences in physiological shape of the axons and dissimilarities in existence of ionic channels [19].

- $\Delta t_{p}$, the IPI between two previous stimuli, i.e., the latest input of the system before arrival of the current stimulus. Normalized amplitude of the response to this input is $A_{p}$.

- $r \in\{0,1\}$, the indicator of the changes in the refractory period of the neuron. When $r=1$, the neuron has received a small gap for recovery after a heavy stimulation and it has not recovered properly, hence, its refractory period has increased [8]. On the other hand, $r=0$ shows that the neuron is recovered properly during previous stimulations and its refractory period is not changed.

- $S$, the state of the axonal memory. Based on experiments on CA region of the brain [8], [9], [16], [18], we define four states for axonal memory as outlined bellow:

1) Resting state, $S=$ Rest : In this state, neuron has received few stimulation during last seconds, hence, it is not depolarized and the amplitude of its axonal response to the first stimulus it receives is around the amplitude of the baseline spike. However, if the neuron receives several stimuli with small IPIs in this state, the amplitude of its axonal response decreases and it passes to the suppression state.

2) Suppression state, $S=S u p:$ In this state, the neuron has received heavy stimulation during last seconds and the amplitude of its response is less than the amplitude of the baseline spike. If the heavy stimulation continues, the neuron stays in this state. However, if the neuron gets some rest, it passes to one of the Fast recovery, Slow recovery, or Resting states depending on the duration of the rest.

3) Fast recovery state, $S=F R e c$ : If the neuron gets a little time to rest after being heavily depolarized in Suppression state, it passes to Fast recovery state. In this state, the response of the neuron is under a fast but frequency-dependent recovery. If the rest time is too small, the neuron cannot recover properly and its refractory period changes.

4) Slow recovery state, $S=S R e c$ : In this state, the axonal response recovery is independent from the intensity of the previous stimulations.

\section{B. Axonal Response}

Next step is defining the relation of the axonal response with axonal input, memory states and parameters. Since neurons may fail to respond a stimulus based on its arrival time [16], we define two parameters, (i) the failure probability, $P_{f}$, and (ii) the normalized amplitude without considering failure, $A_{n}$. Then, the amplitude of the response is calculated as

$$
A=\left\{\begin{array}{ll}
0, & p=P_{f} \\
A_{b} A_{n}, & p=1-P_{f}
\end{array},\right.
$$

where $A_{b}$ is the amplitude of the baseline spike.

To find the axonal response to a stimulus, we have to define functions $F_{P_{f}}($.$) and F_{A_{n}}($.$) such that P_{f}=$
$F_{P_{f}}\left(\Delta t, A_{p}, \Delta t_{p}, r, S\right)$ and $A_{n}=F_{A_{n}}\left(\Delta t, A_{p}, \Delta t_{p}, r, S\right)$. For this aim, we fit curves to experimental data reported in the literature for each state $S$ by minimizing the root mean square error (RMSE), i.e., we select functions $F_{P_{f}}($.$) and F_{A_{n}}($.$) in$ each state such that they minimize the error defined as

$R M S E=\sqrt{\frac{1}{|D|} \sum_{\Delta t \in D}\left(\overline{x\left(\Delta t, A_{p}, \Delta t_{p}, r, S\right)}-x(\Delta t)\right)^{2}}$,

where $D$ is the set of IPIs that their corresponding experimental data is reported in the literature, $|D|$ is the cardinality of set $D, x(\Delta t)$ is the experimental data with IPI $\Delta t$ for failure probability (or normalized amplitude), and $\overline{x(.)}$ is the fitted function to the data provided by $x($.$) , i.e, \overline{x(.)}$ is $F_{P_{f}}($.$\left.) (or F_{A_{n}}().\right)$. The rest of this section contains extracted models from experimental data reported in the literature for the amplitude of the axonal response in each memory state.

1) Resting state: In the experiments that paired pulse stimulation is applied, neuron is not stimulated for a long time before each pair of stimuli. Hence, it is in the Resting state and its response to the first stimulus is the same as baseline spike. However, depending on the IPI, the amplitude of the axonal response to the second stimulus in each pair can be less than the amplitude of the baseline spike. Hence, we use the response to the second stimuli in each pair to model the functionality of the neuron in Resting state.

For IPI less than $2 \mathrm{~ms}$, no response is detected in body temperature, i.e., $37^{\circ} \mathrm{C}$, for both $\mathrm{CA} 1$ and $\mathrm{CA} 3$ pyramidal neurons [16]. Hence, we define $P_{f}=1$ and $A_{n}=0$ for $\Delta t \leq 2 \mathrm{~ms}$. Moreover, from the results of [16], $P_{f}$ and $A_{n}$ vary rapidly with respect to IPI, i.e., $\Delta t$, for $\Delta t>2 \mathrm{~ms}$. Hence, we model their changes with exponential functions as

$$
\begin{gathered}
P_{f}= \begin{cases}1, & \Delta t \leq 0.002 \\
a_{1} \exp \left(-b_{1} \Delta t\right), & \text { otherwise }\end{cases} \\
A_{n}=\left(c_{1}-d_{1} \exp \left(-e_{1} \Delta t\right)\right) u(\Delta t-0.002) .
\end{gathered}
$$

We minimize the RMSE mentioned in (1) to fit (2) and (3) to the experimental data reported in [16] and derive $a_{1}=2$, $b_{1}=357.4 \mathrm{~Hz}, c_{1}=0.95, d_{1}=0.37$, and $e_{1}=80.62 \mathrm{~Hz}$.

Note that a neuron enters Resting state after properly recovering from previously applied stimuli. Hence, $r$ is always zero in Resting state, which shows that the neuron is recovered thoroughly and there is no change in its refractory period.

2) Suppression state: To model functionality of the axon at this state, we refer to experiments with spike train stimulation. The refractory period of the neuron is an important factor to model its functionality in this state. Hence, in the following, we describe axonal functionality for different values of $r$.

a) No change in the refractory period, $r=0$ : No strong suppression occurs on either CA1 or CA3 pyramidal nerve cells under prolonged stimulation with frequencies less than $25 \mathrm{~Hz}$ [9]. However, by applying stimulation with higher frequencies for $1 \mathrm{~min}$, the axonal response depolarizes to different levels. Moreover, the amplitude degradation stops after receiving different number of stimulations for different frequencies [8]. Intensity of firing depends on the number of 
evoked spikes and their amplitude such that multiplication of these two factors during last second of the prolonged HFS is not significantly different for frequencies 50,100 , and $200 \mathrm{~Hz}$ [8]. Hence, we find the maximum amount of suppression in the normalized amplitude, i.e., $A_{\text {end }}$, as a result of applying a spike train stimulation with frequency $f$ by

$$
A_{\text {end }}=\left\{\begin{array}{ll}
c_{2} \Delta t, & \Delta t \leq 0.04 \\
A_{p}, & \text { otherwise }
\end{array},\right.
$$

wehre $\Delta t=\frac{1}{f}$ is the IPI of spikes. Based on the experimental results reported in [8] we select the constant $c_{2}=16 \mathrm{~Hz}$.

To find the axonal response to a spike with IPI $\Delta t$ in Suppression state, we assume that the degradation occurred in axonal response, $A_{p}$, is a result of receiving an spike train with frequency $\frac{1}{\Delta t}$ and the incoming stimulus is $m$ th one in this train. Hence, our task simplifies to finding $m$ and the axonal response to all stimuli during spike train with frequency $\frac{1}{\Delta t}$. According to observations of the axonal response reported in [8], axonal functionality suppresses rapidly among initial seconds of HFS. Hence, we model the axonal response to the $m$ th stimulus in a stimulation train with frequency $\frac{1}{\Delta t}$ by

$$
A_{n}=A_{\text {end }}+\left(1-A_{\text {end }}\right) \exp \left(-a_{2} t_{p_{m}}\right),
$$

where $t_{p_{m}}$ is the total time from the start of the spike train until arrival of the current stimulus and can be written as $t_{p_{m}}=$ $\Delta t+t_{p_{(m-1)}}=m \Delta t$. We can simply substitute $A_{p}$ and $t_{p_{(m-1)}}$ in (5) and derive $t_{p_{(m-1)}}$ as

$$
t_{p_{(m-1)}}=-\frac{1}{a_{2}} \log \frac{A_{p}-A_{\text {end }}}{1-A_{\text {end }}} .
$$

By minimizing the RMSE between respond found by (5) and the experimental data reported in [8], we select $a_{2}=0.62 \mathrm{~Hz}$.

In [18], changes in the failure probability during stimulating CA3 pyramidal neurons with prolong HFS with frequency $100 \mathrm{~Hz}$ is reported. Based on this study, failure probability increases during spike train stimulation. Although no more information exists for failure probability in other frequencies, it is clear that as the stimulus number increases, the failure probability increases. Hence, we model the failure probability in response to $m$ th spike in a train with frequency $\frac{1}{\Delta t}$ by

$$
P_{f}(m)=a_{3}-\frac{a_{3}-P_{f 1}}{\exp (1)} \exp \left(-b_{3}(m-1)\right),
$$

where $a_{3}=0.8$ and $b_{3}=0.05$ are selected to fit these curves to the experimental data reported in [18] by minimizing RMSE. $P_{f 1}$ is the failure probability to the first stimulation of the train and can be calculated by using (2), i.e., the failure probability in response to a stimulus with IPI $\Delta t$ arriving at Resting state. Moreover, $m$ can be derived by substituting $t_{p_{(m-1)}}=(m-1) \Delta t$ in $(6)$.

b) Change in the refractory period, $r=1$ : Based on [8], by inserting a small gap during prolonged HFS, refractory period of the neuron increases. It seems that refractory period increases more for smaller gaps. However, comprehensive information to understand axonal functionality in this situation is not reported in the literature. Hence, to extract a model for these changes and their effects on axonal functionality, more experimental data is needed about response of the neuron after inserting gaps with different durations within spike trains with different frequencies.

3) Fast recovery state: If a neuron is not stimulated for at least $\Delta t_{p}$ second after receiving heavy stimulation and being deeply depolarized, it is in one of recovery states. To find whether the axonal functionality is deeply depolarized, we define a threshold, $A_{t h, F}$, such that $A_{p}<A_{t h, F}$ indicates when the neurons needs Fast recovery. Axonal functionality recovers very fast in this state and the amount of recovery depends on the amount of suppression [8], hence the proposed model should depend on $A_{p}$. Since change in the amplitude after $\Delta t=100 \mathrm{~ms}$ is not significant [8], we ignore it and model the response by

$$
A_{n}= \begin{cases}a_{5}-c_{5} \exp \left(-b_{5}\left(\Delta t-\frac{A_{p}}{c_{2}}\right)\right), & \frac{A_{p}}{c_{2}}<\Delta t<0.1 \\ a_{5}-c_{5} \exp \left(-b_{5}\left(0.1-\frac{A_{p}}{c_{2}}\right)\right), & \text { otherwise }\end{cases}
$$

where $c_{5}=a_{5}-A_{p}$. By minimizing the RMSE between (7) and data reported in [8], we select $a_{5}=0.63$ and $b_{5}=40 \mathrm{~Hz}$.

Although the axonal response does not vary significantly after $\Delta t=100 \mathrm{~ms}$, the neuron needs more time to finish this phase of recovery properly. The duration that a neuron needs to finish Fast recovery is called $T_{s}$. If the recovery time is less than a threshold, which is $\Delta t_{t h}=100 \mathrm{~ms} \ll T_{s}$ based on experimental data reported in [8], the axonal functionality does not recover properly and the refractory period of the neuron increases. In this case, we set the indicator for changes in the refractory period, $r$, to 1 by $r=\left\{\begin{array}{ll}1, & \Delta t_{p}<\Delta t<\Delta t_{t h} \\ 0, & \text { otherwise }\end{array}\right.$.

To become able to model variations in the failure probability during Fast recovery state, more experimental data is needed. The stimulations, needed to be used in these experiments, can be spike trains with different frequencies followed by an spike with IPI greater than $\Delta t_{p}$ and less than $T_{s}$, where the spike train is needed to suppress the axonal response and the purpose of the last spike is entering the neuron to Fast recovery state.

4) Slow recovery state: Based on [8], the amplitude of the evoked spike is recovering slowly and independently from intensity of previous stimulations in this state. The neuron comes to this state in two cases, (i) when its axonal functionality is deeply suppressed prior to current stimulus, i.e., $A<A_{t h, F}$, but IPI of the current stimulus is enough to finish Fast recovery, i.e., $\Delta t>T_{s}$, and start Slow recovery, and (ii) when the axonal functionality is depolarized but not deeply suppressed prior to the current stimulus, i.e., $A_{t h, F} \leq A_{p}<A_{t h}$. In the first case, the neuron spent $T_{s}$ second to finish Fast recovery, hence $\Delta t-T_{s}$ is the time that neuron is in Slow recovery. However, the whole $\Delta t$ is the time that the neuron is in Slow recovery state in the second case. Moreover, the amplitude of the response increases rapidly during the first seconds of the Slow recovery, hence we model it by

$$
A_{n}=\left\{\begin{array}{ll}
1-a_{4} \exp \left(-b_{4}\left(\Delta t-T_{s}\right)\right), & A_{p}<A_{t h, F} \\
1-a_{4} \exp \left(-b_{4} \Delta t\right), & A_{p} \geq A_{t h, F}
\end{array},\right.
$$


where $a_{4}$ shows the maximum amount of recovery and $b_{4}$ is calculated based on $a_{4}$ by defining the time that neuron needs to get enough recovery to enter resting state as $T_{e}$, i.e., by inserting $A_{n}=A_{t h}$ and $\Delta t=T_{e}$ for $A_{p} \geq A_{t h, F}$ or $\Delta t=$ $T_{e}+T_{s}$ for $A_{p}<A_{t h, F}$ in (8) as $b_{4}=-\frac{1}{T_{e}} \log \left(\frac{1-A_{t h}}{a_{4}}\right)$, where $A_{t h}$ is defined as a threshold such that the neuron enters Resting state when $A_{n}$ becomes greater than this threshold. Based on [8], $T_{s}, T_{e}$, and $a_{4}$ do not significantly vary for different intensities of previously applied stimulation. Hence, we select them as $T_{s}=15.9 \mathrm{~s}$ and $T_{e}=56.1 \mathrm{~s}$ for $A_{t h}=$ 0.9 based on the experimental results reported in [8]. If the previous state was Suppression with $r=0$ and $A_{p}<A_{t h, F}$, then we select $a_{4}=0.36$ to minimize the RMSE between (8) and data reported in [8] for axonal recovery after being deeply suppressed. Since in this case, (a) the neuron passes Fast recovery state prior to Slow recovery, (b) the normalized amplitude of the response still needs to be recovered by $a_{4}=$ 0.36 after Fast recovery, and (c) $A_{n}>A_{t h, F}$ indicates that the neuron needs Slow recovery, we define $A_{t h, F}=1-a_{4}=0.64$.

If the previous state of the neuron is Slow recovery state or Suppression with $r=0$ and $A_{p} \geq A_{t h, F}$, then the maximum recovery the neuron needs, i.e., $1-A_{p}$, is less than 0.36 and neuron will continue recovering from $A_{p}$. Hence, we select $a_{4}=\min \left(0.36,1-A_{p}\right)$. Moreover, we set $r$ to zero in this state, which means that neuron is recovered properly.

No information exists in the literature about changes in the failure probability of spikes in this state.

\section{State Transitions}

The axonal memory states and the transition between them are shown in Fig. 3. The rest of this section contains the details of these transitions.

1) Resting state: Based on experiments on hippocampal pyramidal neurons, when the neuron has not stimulated heavily during the last seconds, its response to the stimulation is greater than the case that it has received heavy stimulation. Hence, among memory parameters of the axon, $A_{p}$ indicates whether the neuron is in the first case, which is called Resting state. Thus, by utilizing the experimental data, we define the threshold $A_{t h}$ such that when $A_{p}$ is greater than or equal to it, the neuron is in Resting state. Moreover, when a neuron is in Resting state, it is recovered properly from previously applied stimuli and $r$ is 0 . Hence, if the neuron receives heavy stimulation such that the normalized amplitude of its response, $A_{n}$, becomes less than $A_{t h}$, it passes to Suppression state with $r=0$. Otherwise, it stays in Resting state.

2) Suppression state with $r=0$ : When the neuron is in Suppression state with $r=0$, it can stay in this state or pass to one of the recovery states based on IPI of the stimulus it receives, i.e., $\Delta t$, as stated bellow.

- If $\Delta t \leq \Delta t_{p}$, the neuron stays in Suppression state with $r=0$. In this situation, the amplitude of its response decreases and the probability of failure increases.

- If $\Delta t>\Delta t_{p}$, the recovery of axonal response is started. If the neuron has received heavy stimulations previously, it needs time to finish both Fast and Slow recovery states. Otherwise, Slow recovery state is enough to compensate

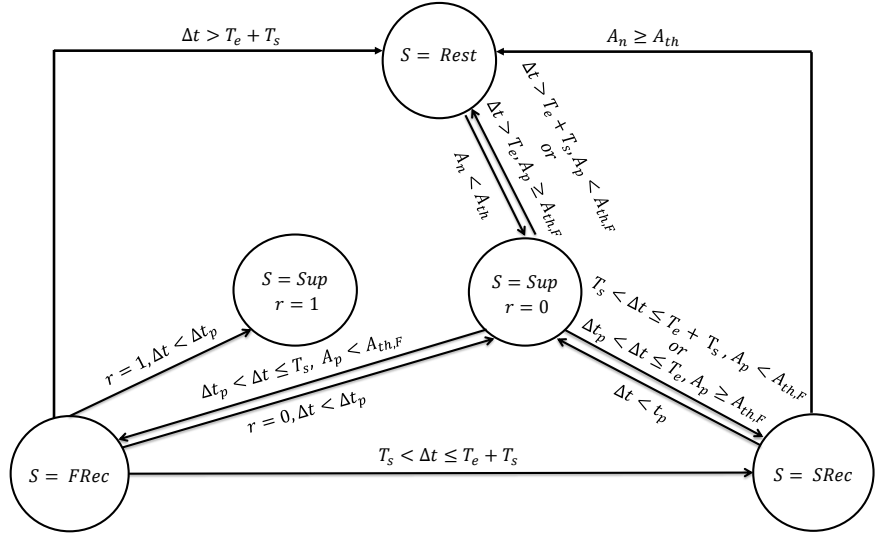

Fig. 3. State diagram of the proposed system for axonal functionality.

the axonal response suppression. $A_{p}$ has information about the intensity of the previously applied stimuli so it indicates whether the neuron needs one or two recovery phases. Hence, both $\Delta t$ and $A_{p}$ are important to find the state that the neuron enters as stated bellow.

a) Fast recovery: The first recovery state after becoming heavily depolarized is fast recovery, which takes $T_{s}$ second to finish. $A_{p}<A_{t h, F}$ shows that the neuron is heavily depolarized and needs to pass Fast recovery state before starting Slow recovery. Hence, if $\Delta t \leq T_{s}$ and $A_{p}<A_{t h, F}$, the response of the neuron to current stimulation is based on Fast recovery state.

b) Slow recovery: The neuron enters this state under two situations: (i) the neuron is not heavily depolarized prior to the arrival of the current stimulus, i.e., $A_{P} \geq$ $A_{t h, F}$, and the IPI of the current stimulus is not enough to finish Slow recovery state and enter Resting state, i.e., $\Delta t \leq T_{e}$, (ii) the neuron is heavily depolarized before current stimulus, i.e., $A_{P}<A_{t h, F}$, and it has enough time to finish Fast recovery but not Slow recovery state, i.e., $T_{s}<\Delta t \leq T_{e}+T_{s}$.

c) Resting: If the neuron has enough time to finish Slow recovery in each of the aforementioned situations that it needs to pass Slow recovery state, i.e., (i) $\Delta t>T_{e}$ and $A_{P} \geq A_{t h, F}$ or (ii) $\Delta t>T_{e}+T_{s}$ and $A_{P}<A_{t h, F}$, the axonal recovery is finished and the response to the current stimulus is based on Resting state.

3) Fast recovery state: The possible transitions from Fast recovery state based on the IPI of the current stimulus, i.e., $\Delta t$, are as follows.

- If $\Delta t \leq \Delta t_{p}$, the neuron passes to suppression state.

- If $\Delta t>\Delta t_{p}$, the axonal functionality is recovering and the axonal response is based on one of following states.

a) Fast recovery: If the neuron does not receive enough time to finish Fast recovery, i.e., $\Delta t_{p}<\Delta t \leq T_{s}$, the neuron stays in Fast recovery state.

b) Slow recovery: If the IPI of the current stimulus is enough to finish Fast recovery bot not Slow recovery, i.e., $T_{s}<\Delta t \leq T_{e}+T_{s}$, next state is Slow recovery.

c) Resting: If the neuron receives enough time to recover the axonal functionality, i.e., $T_{e}+T_{s}<\Delta t$, the neuron 


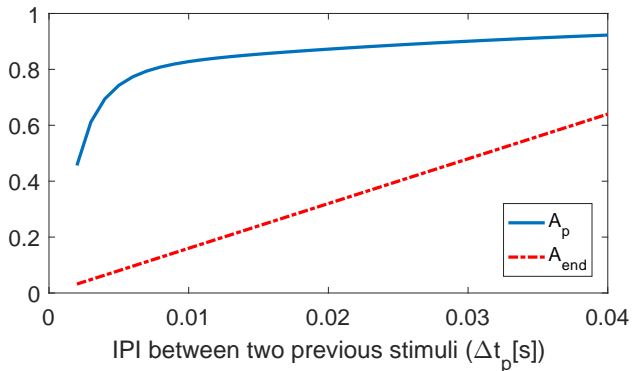

Fig. 4. Comparing the worst case of $A_{p}$ and $A_{\text {end }}$ in the Suppression state when the previous state of the axon is Resting.

goes to Resting state.

4) Slow recovery state: When a neuron is in Slow recovery state, it can pass to Suppression state with $r=0$ or stay in Slow recovery state as stated bellow.

- If $\Delta t \leq \Delta t_{p}$, the neurons passes to the Suppression state while its refractory period is not changed, i.e., $r=0$.

- If $\Delta t>\Delta t_{p}$, the neuron stays in Slow recovery state. In this case, if the normalized amplitude of the response reaches $A_{t h}$ the recovery is finished and the neuron goes to Resting state.

\section{Validation of the transition among states}

Last step in defining a system model for axonal functionality is verifying that the model meets following conditions.

a) If the neuron enters or stays in Resting state, the normalized amplitude of the axonal response must be greater than $A_{t h}$.

b) If the neuron enters or stays in Suppression state, normalized amplitude of the axonal response must not increase.

c) If the neuron enters or stays in Fast recovery state, the normalized amplitude of the axonal response, $A_{n}$, must not decrease and it must be less than $A_{t h, F}$.

d) If the neuron enters or stays in Slow recovery state, the normalized amplitude of the axonal response, $A_{n}$, must not decrease and it must be less than $A_{t h}$.

Since a neuron can enter each of the memory states from different states as shown in Fig. 3, we list all of the possible cases to enter a state and show that the aforementioned conditions are valid in the defined system.

1) Resting state: We compare $A_{n}$ and $A_{t h}$ for all cases of entering Resting state from other states as listed bellow.

- The previous state is Resting or Slow recovery state: The neuron enters Resting state from each of the Slow recovery and Resting states when the normalized amplitude of the axonal response, $A_{n}$, is greater than or equal to $A_{t h}$.

- The previous state is Suppression with $r=0$ or Fast recovery: The neuron enters Resting state from these two states when IPI of the current stimulus, i.e., $\Delta t$, is enough to finish the recovery of axonal functionality. Hence, the axonal response to the current stimulus is based on Resting state with $\Delta t>T_{e}$, where $T_{e}=56.1 \mathrm{~s}$. Thus, the normalized amplitude of the axonal response, $A_{n}$, is greater than $A_{t h}$ based on (3).

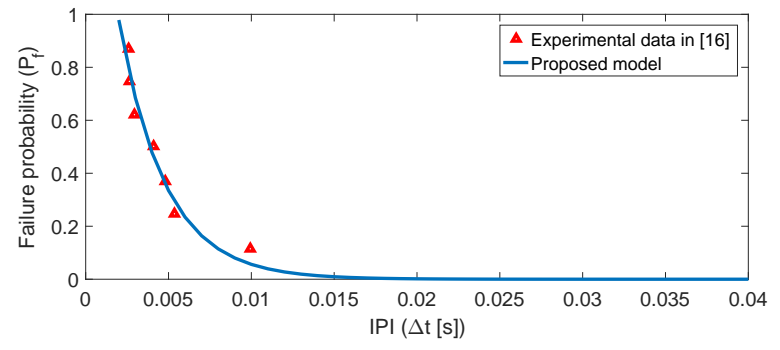

(a)

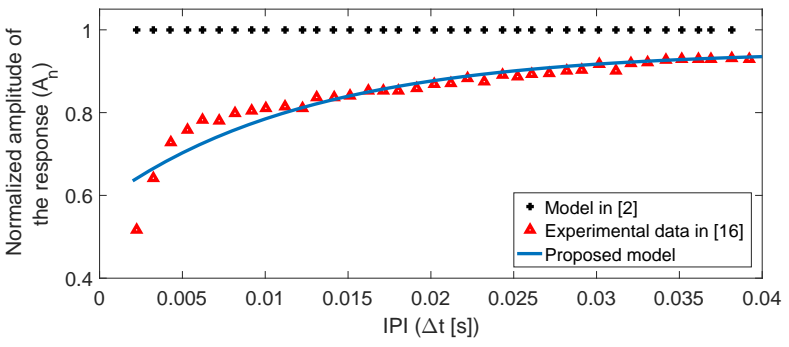

(b)

Fig. 5. (a) Failure probability and (b) normalized amplitude of the response for different arrival times of the second stimulus in a pair when the neuron is in Resting state based on experimental results in [16] and discussed models.

2) Suppression state with $r=0$ : We analyze the performance of this state by checking whether its axonal response suppresses independent from its previous state. Since $A_{\text {end }}$ shows the maximum suppression if we apply a train of stimulation with frequency $\frac{1}{\Delta t}$, if $A_{\text {end }}$ is less than $A_{p}, A_{n}$ is also less than $A_{p}$. Hence, we compare $A_{\text {end }}$ and $A_{p}$ for entering Suppression state with $r=0$ from following states.

- Resting state: Since $\Delta t \leq \Delta t_{p}$ and $A_{\text {end }}$ is calculated based on (4), the highest possible value of $A_{\text {end }}$ is occurring when $\Delta t=\Delta t_{p} . A_{p}$ and $A_{\text {end }}$ for different values of $\Delta t_{p}$ are shown in Fig. 4. Based on this figure, for all possible values for $\Delta t_{p}, A_{\text {end }}$ is less than $A_{p}$.

- Suppression state with $r=0$ : Hence, $A_{p} \geq c_{2} \Delta t_{p}$. To stay at Suppression state $\Delta t$ should not be greater than $\Delta t_{p}$. Hence, $A_{\text {end }} \leq A_{p}$ based on (4).

- Slow recovery state: Based on (8), $A_{p} \geq A_{t h, F}=0.64$, and based on (4), $A_{\text {end }} \leq 0.64$, hence, $A_{\text {end }} \leq A_{p}$.

- Fast recovery state: Since $r$ is equal to $0, \Delta t_{p}$ should be greater than $100 \mathrm{~ms}$ and based on the fact that in the Fast recovery state change in the amplitude from $\Delta t_{p}=100 \mathrm{~ms}$ is not significant, $A_{p}$, is around 0.61 . By comparing $A_{\text {end }}$ given by (4) and $A_{p}=0.61$, when $0.038<\Delta t<0.04$ we have $A_{\text {end }}>A_{p}$. However, the difference of $A_{\text {end }}$ and $A_{p}$ is small, less than 0.03, and the amplitude of the response will increase slightly, which can always happen in the real scenarios.

Hence, in most of the cases in this state, $A_{\text {end }}$ is less than or equal to $A_{p}$, which means that the normalized amplitude of the axonal response, i.e., $A_{n}$, is not increasing.

3) Fast recovery: The only possible transitions to the Fast recovery state are from Suppression state with $r=0$ or Fast recovery state. In both of these cases, the normalized amplitude of the response is calculated based on (7), where the normalized amplitude is always between $A_{p}$ and $A_{t h, F}$. 


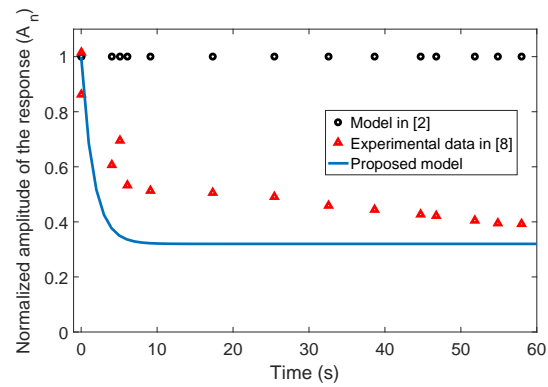

(a) Frequency $50 \mathrm{~Hz}$

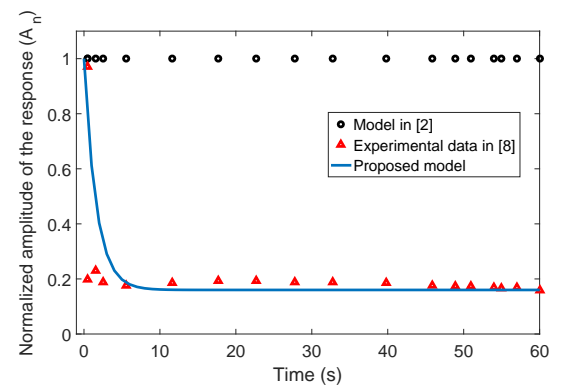

(b) Frequency $100 \mathrm{~Hz}$

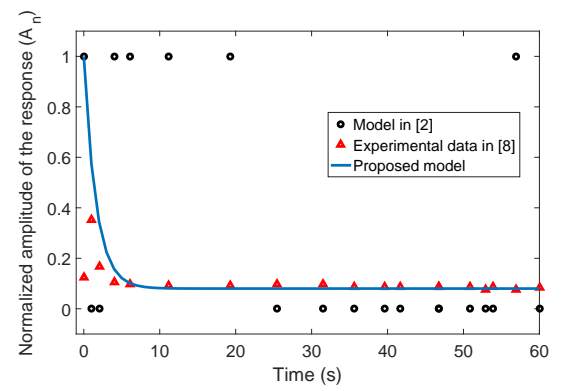

(c) Frequency $200 \mathrm{~Hz}$

Fig. 6. Normalized amplitude of the response to HFS for different frequencies in the Suppression state based on the proposed model, existing model in [2] and experimental data in [8].

Moreover, $A_{p}$ is less than $A_{t h, F}$ in both of the aforementioned transitions. Hence, the normalized amplitude of the response, $A_{n}$ is less than $A_{t h, F}$ and not decreasing.

4) Slow recovery: The neuron can stay in this state or enter it from Fast recovery state or Suppression state with $r=0$. Here, we analyze the axonal response in each of these cases.

- The neuron stays in Slow recovery state if the normalized amplitude of its response, $A_{n}$, is less than $A_{t h}$. Moreover, since the axonal response is calculated based on (8), its normalized value, i.e., $A_{n}$, is not decreasing.

- In both of aforementioned cases of entering the Slow recovery state from other states, the normalized amplitude of its response to the current stimulus is calculated based on (8). Moreover, the IPI of the current stimulus, $\Delta t$, is less than the time that neuron needs to enter Resting state. Hence, the normalized amplitude of the response, $A_{n}$, is less than $A_{t h}$ and not decreasing.

\section{REsults}

In this section, we compare results of the proposed model with data reported in the literature from experiments on hippocampal pyramidal neurons and the results of modeling axonal transmission with Butterworth filter [2]. For this aim,

- We evaluate the performance of proposed memory states by evaluating the RMSE between models and experimental data reported in the literature for normalized amplitudes and failure probabilities in each of states.

- We calculate the response of the overall system to a sample input, which is extracted from physiology literature and contains all of the defined states, to evaluate the accuracy of transitions among states.

\section{A. Performance of memory states}

Axonal model of [2] does not contain aforementioned states. To compare the results of our proposed states with this work,

- We apply inputs according to the Resting and Suppression states to this model, and consider its response for Resting and Suppression states, respectively.

- Since there is no Suppression state in this model, we cannot extract meaningful response for recovery states.

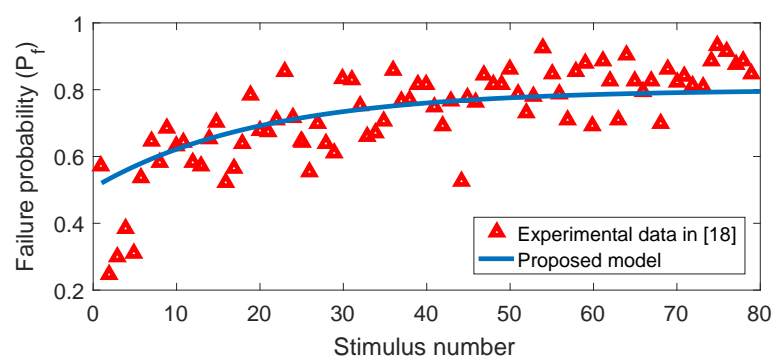

Fig. 7. Failure probability in Suppression state as a function of stimulus number at frequency $100 \mathrm{~Hz}$ based on proposed model and experimental data in [18].

1) Response in Resting state: To evaluate the performance of our proposed models for Resting state, we consider memory parameters as $A_{p}=1, \Delta t_{p}=0$, and $\Delta t \gg T_{e}$ and give paired pulse stimuli with different IPI as the input of the models. The response to the first stimulus of each pair is the same as the baseline spike. The response to the second stimulus in a pair depends on the IPI.

As it is depicted in Fig. 5, the response of the existing model in [2] to these stimulations is always 1, and changes in the normalized amplitude of the response are not considered. On the other hand, our proposed model reaches a good estimation with RMSE equal to 0.05 and 0.03 for the failure probabilities and normalized amplitudes, respectively.

2) Response in Suppression state with $r=0$ : To measure the performance of this state, we give trains of stimuli with frequencies $50 \mathrm{~Hz}, 100 \mathrm{~Hz}$, and $200 \mathrm{~Hz}$ as the input of the models and check the axonal response variations during spike train. We select these frequencies because response of the axon to these stimulations exists in the literature.

Normalized amplitude of the response for these frequencies is depicted in Fig. 6 based on the models and experimental data in [8]. We can see that the existing model in [2] cannot be used to accurately model the axonal response while it is in Suppression state, but our proposed model follows amplitude variations properly. The RMSE between the proposed model and experimental data reported in [8] for frequencies $50 \mathrm{~Hz}$, $100 \mathrm{~Hz}$, and $200 \mathrm{~Hz}$ are $0.17,0.07$, and 0.06 , respectively.

Failure probability for different stimulus numbers in a train with frequency $100 \mathrm{~Hz}$ based on our model and experimental data in [18] is shown in Fig.7 and the RMSE between our 


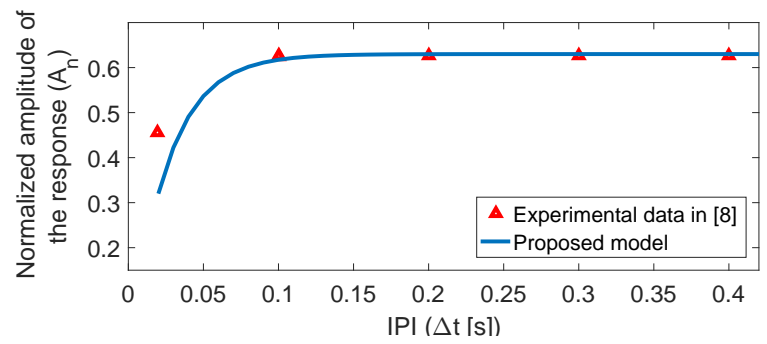

(a)

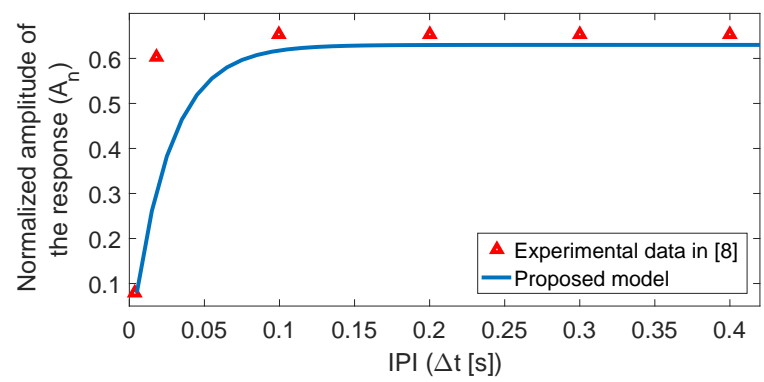

(b)

Fig. 8. Normalized amplitude of the response in Fast recovery state based on proposed model and experimental data in [8]. In experimental data, to suppress neuron before fast recovery, a spike train with frequency (a) $50 \mathrm{~Hz}$ and (b) $200 \mathrm{~Hz}$ is applied to it. In the model, $A_{p}=\frac{16}{f}$ shows amount of suppression before recovery phase, which is selected as (a) 0.32 and (b) 0.08 .

model and these experimental data is 0.09 .

3) Response in Fast Recovery state: To study the axonal functionality during the fast recovery, firstly, spike train stimulations are applied to the neuron in the physiology literature to suppress them. Then, a stimulus with different arrival time according to the fast recovery is applied to the neuron [8]. On the other hand, in the Suppression state of the proposed model, maximum amount of the suppression as a result of receiving a spike train is calculated based on (4). Hence, we select $A_{p}=\frac{16}{f}$ for different frequencies of the previously applied spike trains in the experiments and find the normalized amplitude of axonal response, $A_{n}$, based on our model.

The normalized amplitude of the response during fast recovery is depicted in Fig. 8. The RMSE between model and experimental data reported in [8] is 0.09 in this state.

4) Response in Slow Recovery state: The processes used in the physiology literature to study the axonal functionality during slow and fast recovery are the same [8]. Hence, to measure the performance of our model in this state, we use the same procedure as Fast recovery state, i.e. we set $A_{p}=\frac{16}{f}$. Then, we give stimuli with different IPI as input of our model.

Normalized amplitude of the response to stimuli with different IPI is depicted in the Fig. 9. The overall RMSE between the model and experimental data reported in [8] is 0.03 .

\section{B. Performance of the transitions among memory states}

Based on the simulation results provided in the previous section, the proposed model for each of the memory states follows the axonal functionality properly. In this section, we study the accuracy of the transition between states and the overall model by considering stimulations that contain all of

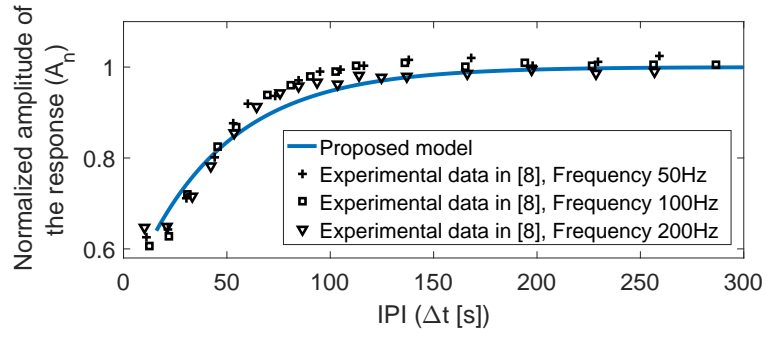

Fig. 9. Normalized amplitude of the neuron response in Slow recovery state to stimulations with different time intervals. Neuron is suppressed due to receiving stimulation train with different frequencies prior to last stimulus.

the defined states and the response of the neuron to them is reported in the literature. Each stimulation contains (i) $1 \mathrm{~min}$ spike train with frequency $100 \mathrm{~Hz}$, in Fig. 10 (a), or $200 \mathrm{~Hz}$, in Fig. 10(b), and (ii) 1 stimulus with different arrival times after spike train termination.

The axon is at rest before receiving first stimulus, hence, the response to the first stimulus is calculated based on the Resting state. After some stimuli, the amplitude of the response decreases and become less than $A_{t h}$, which is set to 0.9 in our model. At this time, axon goes to the Suppression state. After 1 min stimulation with spike train, only one stimulus is given to the axon with different IPIs. If IPI of this stimulus is less than $T_{s}$, then the response is based on Fast recovery state. Otherwise, the response is based on the Slow recovery state.

In [2], the axon is modeled with a low-pass filter. Hence, the amplitude of its response to the spike train with frequency $100 \mathrm{~Hz}$ is always 1, as it is depicted in Fig. 10(a). However, when the frequency of the spike train increases to $200 \mathrm{~Hz}$, the input goes to the transition band of the low-pass filter and because of using a spike detector after low-pass filter in the model, the amplitude of its response varies between 1 and 0 as depicted in Fig. 10(b). Since many samples exist in 1 min stimulation with frequency $200 \mathrm{~Hz}$, we select and plot some of them to reach a clear figure in Fig. 10(b).

It is clearly shown in Fig. 10 that our model accurately captures the variation of the spike amplitude during axonal transmission, which was missing in the exiting literature [2].

\section{DISCUSSION}

The existing studies in the literature on modeling neurospike communication focus on synaptic communication and consider the amplitude of the spike during axonal transmission as all or none. However, the shape of the spike during axonal transmission may vary, which has a direct relation with the probability of error detection in neuro-spike communication. Hence, these changes should be considered to propose a proper model for functionality of neurons. In this work, we modeled axonal functionality based on the experimental data reported in the literature. We defined 4 states to find amplitude of the spike during axonal transmission, namely Resting, Suppression, Slow recovery and Fast recovery. Then, we proposed realistic models to find amplitude and failure probabilities of the response in these states. Based on the results of the simulations, we observed that the proposed 


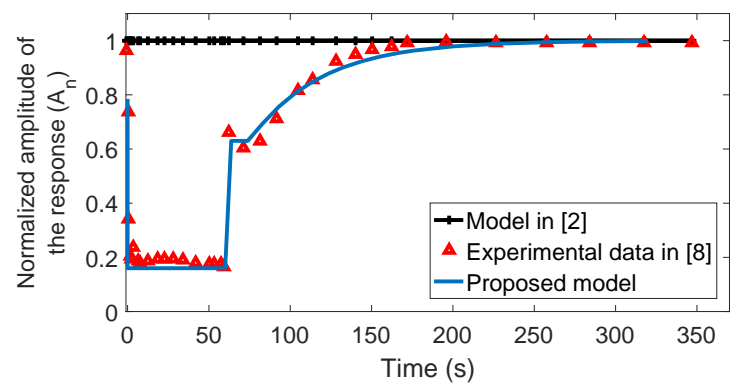

(a) Frequency of spike train $=100 \mathrm{~Hz}$.

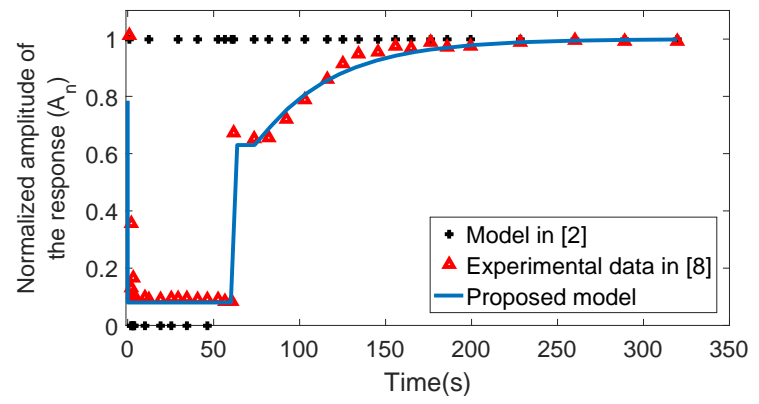

(b) Frequency of spike train $=200 \mathrm{~Hz}$.

Fig. 10. Normalized amplitude of the response of the proposed model, mode in [2], and experimental data in [8] to the sample stimulations, which contain 1 min train stimuli and 1 stimulus with different arrival times after that.

model follows axonal functionality properly. However, more experimental data is needed to evaluate the proposed model, for instance, reports on the exact rate of degradation and its stop time at different frequencies lead to more accurate models for Suppression state, and complete the following parts:

- Variation in the failure probability during Suppression, Fast and Slow recovery states when refractory period is not changed.

- Changes in the refractory period after inserting a small gap during HFS, and the effect of these changes on the axonal functionality in Suppression state with $r=1$, Fast and Slow recovery states.

To complete the proposed model for axonal functionality, changes in the width and delay of spikes should also be studied. Changes in the width of spikes during Suppression state for hippocampal granule cells, which send information to CA3 pyramidal neurons, is reported in [10]. On these neurons, the width of spike can increase to 3 times, which is affecting the release of vesicles to the synapse and the probability of error detection in neuro-spike communication. On the other hand, changes in the propagation delay of spikes might introduce some noise into the coding of neural information in the brain [20]. Studying these parts also needs more experimental data.

\section{REFERENCES}

[1] I. F. Akyildiz et al., "Nanonetworks: A new communication paradigm," Computer Networks, vol. 52, no. 12, pp. 2260-2279, 2008.

[2] E. Balevi and O. B. Akan, "A physical channel model for nanoscale neuro-spike communications," IEEE Trans. Commun., vol. 61, no. 3, pp. 1178-1187, 2013.

[3] O. B. Akan et al., "Fundamentals of molecular information and communication science," Proc. IEEE, 2016.
[4] D. Malak and O. B. Akan, "A communication theoretical analysis of synaptic multiple-access channel in hippocampal-cortical neurons," IEEE Trans. Commun., vol. 61, no. 6, pp. 2457-2467, 2013.

[5] A. Manwani and C. Koch, "Detecting and estimating signals over noisy and unreliable synapses: information-theoretic analysis," Neural computation, vol. 13, no. 1, pp. 1-33, 2001.

[6] L. Galluccio et al., "Modeling signal propagation in nanomachine-toneuron communications," Nano Communication Networks, vol. 2, no. 4, pp. 213-222, 2011.

[7] T. Sasaki, "The axon as a unique computational unit in neurons," Neuroscience research, vol. 75, no. 2, pp. 83-88, 2013.

[8] Z. Feng et al., "High frequency stimulation extends the refractory period and generates axonal block in the rat hippocampus," Brain stimulation, vol. 7, no. 5, pp. 680-689, 2014.

[9] A. L. Jensen and D. M. Durand, "High frequency stimulation can block axonal conduction," Experimental neurology, vol. 220, pp. 57-70, 2009.

[10] J. R. Geiger and P. Jonas, "Dynamic control of presynaptic ca 2+ inflow by fast-inactivating $\mathrm{k}+$ channels in hippocampal mossy fiber boutons," Neuron, vol. 28, no. 3, pp. 927-939, 2000.

[11] D. Debanne et al., "What are the mechanisms for analogue and digital signalling in the brain?" Nature Reviews Neuroscience, vol. 14, no. 1 , pp. 63-69, 2013.

[12] T. Sasaki et al., "Action-potential modulation during axonal conduction," Science, vol. 331, no. 6017, pp. 599-601, 2011.

[13] H. Ramezani and O. B. Akan, "Synaptic channel model including effects of spike width variation," in Proc. NANOCOM. ACM, 2015, p. 11.

[14] J. P. Meeks et al., "Action potential fidelity during normal and epileptiform activity in paired soma-axon recordings from rat hippocampus," The Journal of physiology, vol. 566, no. 2, pp. 425-441, 2005.

[15] A. Soleng et al., "Activity-dependent excitability changes in hippocampal ca3 cell schaffer axons," The Journal of physiology, vol. 560, no. 2, pp. 491-503, 2004.

[16] M. Raastad and G. M. Shepherd, "Single-axon action potentials in the rat hippocampal cortex," The Journal of physiology, vol. 548, no. 3, pp. 745-752, 2003.

[17] Z. Feng et al., "Functional disconnection of axonal fibers generated by high frequency stimulation in the hippocampal cal region in-vivo," Brain research, vol. 1509, pp. 32-42, 2013.

[18] E. Kim et al., "Decreased afferent excitability contributes to synaptic depression during high-frequency stimulation in hippocampal area ca1," Journal of neurophysiology, vol. 108, no. 7, pp. 1965-1976, 2012.

[19] B. P. Bean, "The action potential in mammalian central neurons," Nature Reviews Neuroscience, vol. 8, no. 6, pp. 451-465, 2007.

[20] D. Debanne, "Information processing in the axon," Nature Reviews Neuroscience, vol. 5, no. 4, pp. 304-316, 2004.

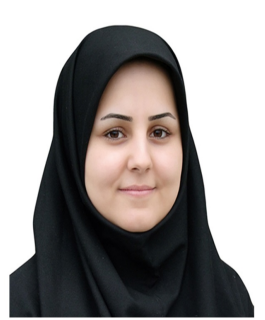

Hamideh Ramezani [S'15] received her B.S. degree in Electrical Engineering, the major of Communication from University of Tehran, Iran in 2011 and M.S. degree in Electrical Engineering, the major of Communication Systems from Sharif University of Technology, Iran in 2013. She is currently a research assistant in the Next-generation and Wireless Communications Laboratory (NWCL) and pursuing her Ph.D. degree in Electrical and Electronics Engineering at Koc University, Istanbul, Turkey. Her current research interests include molecular communications and intra-body nanonetworks.

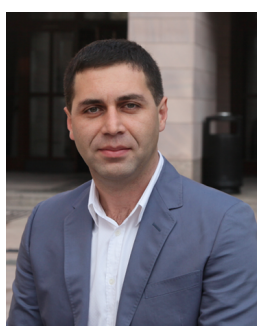

Ozgur B. Akan [M'00-SM'07-F'16] received PhD degree from the School of Electrical and Computer Engineering, Georgia Institute of Technology, USA, in 2004. He is currently with the Electrical Engineering Division, Department of Engineering, University of Cambridge, UK and the Department of Electrical and Electronics Engineering, Koc University, Istanbul, Turkey. His research interests include wireless, nano, molecular communications, and Internet of Everything. 